\title{
Investigating the efficacy of pamidronate, a chemical inhibitor of farnesyl pyrophosphate synthase, in the inhibition of influenza virus infection in vitro and in vivo
}

\author{
KAI SEN TAN ${ }^{1,2}$, WAI CHII NG ${ }^{1}$, JU EE SEET $^{3}$, FARZAD OLFAT ${ }^{2}$, \\ BEVIN P. ENGELWARD ${ }^{2,4}$ and VINCENT T.K. CHOW $^{1,2}$
}

\begin{abstract}
${ }^{1}$ Host And Pathogen Interactivity Laboratory, Department of Microbiology, Yong Loo Lin School of Medicine, National University of Singapore, Singapore $117545 ;{ }^{2}$ Infectious Diseases Interdisciplinary Research Group, Singapore-Massachusetts Institute of Technology Alliance for Research and Technology, Singapore 138602;

${ }^{3}$ Department of Pathology, National University Hospital, Singapore 119074, Republic of Singapore;

${ }^{4}$ Department of Biological Engineering, Massachusetts Institute of Technology, Cambridge,
\end{abstract} MA 02139, USA

Received June 6, 2013; Accepted September 20, 2013

DOI: $10.3892 / \mathrm{mmr} .2013 .1750$

\begin{abstract}
Influenza A virus has caused significant pandemics in the past decades, including the H1N1-2009 pandemic. Viperin is an interferon-inducible protein that acts as a broad-spectrum antiviral protein via the inhibition of farnesyl pyrophosphate synthase (FPPS). To mimic this activity of viperin, the present study investigated the effectiveness of a commercially available FPPS inhibitor (pamidronate) as an inhibitor of influenza virus infection in vitro and in vivo. HeLaM cells were treated with pamidronate to determine its effect on the replication of influenza virus A/H1N1/WSN/1933. C57BL/6 mice were also subjected to intratracheal pamidronate treatment regimes prior to and following lethal influenza challenge. Treatment with the FPPS inhibitor in vitro resulted in a considerable reduction in the viral titer of $\sim 1 \log$ and diminished lipid raft formation without cellular toxicity, thus mimicking the antiviral effect of viperin. However, pamidronate lacked efficacy in vivo and was associated with increased pulmonary damage, most likely due to the complexity of drug-host interactions in the infected mice. Further studies are warranted on pamidronate treatment in other infectious diseases that are more susceptible to FPPS inhibition.
\end{abstract}

Correspondence to: Dr Vincent Chow, Host And Pathogen Interactivity Laboratory, Department of Microbiology, Yong Loo Lin School of Medicine, National University Health System, National University of Singapore, 5 Science Drive 2, Kent Ridge, Singapore 117545, Republic of Singapore

E-mail: micctk@nus.edu.sg

Key words: influenza, bisphosphonate, pamidronate, farnesyl pyrophosphate synthase, lipid rafts, viperin, prophylaxis

\section{Introduction}

Influenza has resulted in large numbers of vaccine-preventable deaths worldwide (1). For example, the Spanish influenza pandemic in 1918 alone caused a large number of fatalities (estimated up to 40 million) within a short period (2). Furthermore, with the highly infectious H1N1-2009 strain and the highly virulent $\mathrm{H} 5 \mathrm{~N} 1$ and $\mathrm{H} 7 \mathrm{~N} 9$ strains of recent years, influenza virus infection remains a major health threat to humankind $(3,4)$.

An emerging strategy in antiviral discovery is to exploit the host innate antiviral mechanisms to counter viral infections. This approach, as it is independent of viral mutation, allows universal targeting of the virus since there are generally no direct interactions with viral proteins (5-8). An example of such a factor is viperin, which is highly induced by both type I and II interferons in response to lipopolysaccharide and double-stranded RNA $(9,10)$. Viperin interacts with farnesyl pyrophosphate synthase (FPPS), which is an enzyme that catalyzes the formation of farnesyl pyrophosphate, an isoprenoid metabolic pathway intermediate. Cholesterol formation is thus reduced, which subsequently disrupts raft assembly and perturbs lipid raft formation (11-13).

Since influenza virus requires lipid rafts for its replication, including viral entry, assembly and budding $(14,15)$, we therefore hypothesized that influenza virus is susceptible to FPPS inhibition. An in vitro study demonstrated that the ectopic expression of viperin successfully inhibits influenza virus budding from cells, leading to reduced virus titers (11). FPPS is also inhibited by nitrogen-containing bisphosphonates (N-bisphosphonates), a family of FPPS inhibitors used clinically in the treatment of bone diseases $(16,17)$. These FPPS inhibitors act by recognizing and binding to bisphosphonate-binding sites on the FPPS enzyme, thereby inactivating it (18). Therefore, we hypothesized that $\mathrm{N}$-bisphosphonates prevent the formation of lipid rafts by mimicking the mechanism of viperin. 
The present study investigated a potential treatment for influenza infection using disodium pamidronate, otherwise known as Pamisol ${ }^{\circledR}$ (PAM), a clinically approved N-bisphosphonate. The study tested the efficacy of this commercially available FPPS inhibitor as an alternative to recombinant viperin protein in influenza therapy. The rationale underlying this study is to leverage on novel host antiviral factors and responses to mitigate influenza infection.

\section{Materials and methods}

Virus and cell cultures. The influenza virus strain A/H1N1/WSN/1933 (WSN33; American Type Culture Collection, Manassas, VA, USA) was employed. MDCK cells (American Type Culture Collection) were cultured in Eagle's minimal essential medium with $10 \%$ fetal bovine serum (FBS). HeLaM cells (American Type Culture Collection) were cultured in Dulbecco's modified Eagle's medium (DMEM) with $10 \%$ FBS.

Drug treatment and animals. All experiments involving treatment of C57BL/6 mice with PAM (Hospira, Inc., Lake Forest, IL, USA) were approved by the Institutional Animal Care and Use Committee (IACUC), National University of Singapore (protocol 050/11).

Infection and treatment of cells. Overnight cultures of $1 \times 10^{5} \mathrm{HeLaM}$ cells were infected with $1 \mu \mathrm{g} / \mathrm{ml}$ TPCK-trypsin-activated WSN33 virus at an MOI of 0.5 , and incubated at $37^{\circ} \mathrm{C}$ for $1 \mathrm{~h}$. The viral inoculum was replaced with $500 \mu \mathrm{l}$ DMEM containing 100,500 or 1,000 $\mu \mathrm{M}$ PAM, or with DMEM containing PBS as control. Following incubation at $37^{\circ} \mathrm{C}$ for $48 \mathrm{~h}$, each cell culture supernatant was harvested for determination of the viral titer. Quantification of the viral titer was determined by a virus plaque assay, as previously described (19).

Cell viability assay. Overnight cultures of $1 \times 10^{5} \mathrm{HeLaM}$ cells in 96-well plates were treated with DMEM containing $1,000 \mu \mathrm{M}$ PAM or PBS, while DMEM alone served as a control. Following incubation at $37^{\circ} \mathrm{C}$ for $48 \mathrm{~h}, 20 \mu \mathrm{l}$ pre-mixed 3-(4,5-dimethylthiazol-2-yl)-5-(3-carboxymethoxyphenyl)2-(4-sulfophenyl)-2H-tetrazolium inner salt (MTS) reagent (Promega, Madison, WI, USA) was added to each well. After incubation at $37^{\circ} \mathrm{C}$ for $3 \mathrm{~h}$, the absorbance readings at $490 \mathrm{~nm}$ of each well were obtained using an ELISA plate reader (Infinit M200; Tecan, Männedorf, Switzerland) to assess whether the PAM treatment had detrimental effects.

Cholera toxin subunit-B (CTxB) staining for lipid rafts. Overnight cultures of $1 \times 10^{5} \mathrm{HeLaM}$ cells were infected with $1 \mu \mathrm{g} / \mathrm{ml}$ TPCK-trypsin-activated WSN33 virus at an MOI of 0.5 . Following incubation at $37^{\circ} \mathrm{C}$ for $1 \mathrm{~h}$, the viral inoculum was replaced with DMEM containing 1,000 $\mu \mathrm{M}$ PAM, or with DMEM containing PBS as control. Following incubation at $37^{\circ} \mathrm{C}$ for 24 or $48 \mathrm{~h}$, the cells were trypsinized, washed and resuspended in chilled DMEM with $10 \%$ FBS. The cells were centrifuged at 1,200 $\mathrm{x}$ g for $5 \mathrm{~min}$ and resuspended in $2 \mathrm{ml}$ of $1 \mu \mathrm{g} / \mathrm{ml} \mathrm{CTxB}$ fluorescent conjugate (Molecular Probes, Camarillo, CA, USA) in complete medium, and incubated at $4^{\circ} \mathrm{C}$ for $10 \mathrm{~min}$. The cells were then washed and fixed in $4 \%$ formaldehyde at $4^{\circ} \mathrm{C}$ for $15 \mathrm{~min}$, and washed again. The cells were cyto-centrifuged onto microscopy slides at $600 \mathrm{x} g$ for 5 min and exposed to mounting medium with DAPI (Vector Laboratories, Burlingame, CA, USA). Using a fluorescence microscope (BX60; Olympus, Tokyo, Japan), 10 random fields were selected for each slide and the mean percentage of CTxB-positive cells was calculated for analysis of the lipid raft expression levels in the cells.

In vivo treatment with an FPPS inhibitor (PAM). Ten-week old C57BL/6 mice were divided into four groups and weighed prior to the experiments. For the prophylactic (PRO) treatment regime, mice were anesthetized with a mixture of $7.5 \mathrm{mg}$ ketamine and $0.1 \mathrm{mg}$ medetomidine, and given a $50 \mu \mathrm{l}$ dose of $5 \mathrm{mg} / \mathrm{kg}$ PAM via the intratracheal route one day prior to infection (day -1). The day after the first treatment (day 0), a second $50 \mu$ l dose of $5 \mathrm{mg} / \mathrm{kg}$ PAM pre-mixed with a lethal dose of WSN33 virus $(1,000 \mathrm{pfu})$ was administered via the intratracheal route. For the post-exposure prophylaxis (PEP) regime, mice were infected with 1,000 pfu of WSN33 virus and then subjected to a $50-\mu 1$ dose of $5 \mathrm{mg} / \mathrm{kg}$ PAM on days 0,1 and 2. Infected mice were monitored daily for their weight and clinical condition, and were promptly euthanized in a $\mathrm{CO}_{2}$ chamber when they reached the end point of either $30 \%$ weight loss, moribund condition or at day 14 in compliance with IACUC guidelines. Following euthanasia, the lungs of the mice were harvested for further processing as previously described (19), where $10 \%$ of the total lung mass was used for virologic and molecular analyses, while the remaining $90 \%$ was for histopathology.

Histopathological staining of murine lungs. The remaining $90 \%$ of each lung was fixed in $4 \%$ formalin, and stained with hematoxylin and eosin (H\&E) as previously described (19). The scoring of lung damage was performed by an experienced pulmonary histopathologist (J. E. Seet), based on a modified scoring system (20), i.e. lung damge score = percentage significant lung damage $\mathrm{x}$ [alveolar hemorrhage +2 (alveolar inflitrates) +3 (fibrin) + alveolar septal congestion]. Each of the four criteria was scored on a scale of 0-3. The scoring was based on the worst-affected areas of the lungs. The percentages of the significantly damaged regions (defined as at least two criteria with a maximum score of 3 , with the whole lung considered as $100 \%$ ) were determined.

Quantitative PCR ( $q P C R)$ and statistical analyses. RNA extraction and qPCR were performed as previously described (19). Statistical analyses were performed using SPSS software, version 19 (SPSS, Inc., Chicago, IL, USA) and GraphPad Prism, version 5 (GraphPad Software, Inc., La Jolla, CA, USA), via Student's t-test for comparison of means, and Kaplan-Meier $\log$ rank survival analysis for survival data. Results were represented as the mean \pm standard deviation. $\mathrm{P}<0.05$ was considered to indicate a statistically significant difference.

\section{Results}

PAM treatment reduces viral titer and has no detrimental effect on cell viability in vitro. Treatment of infected HeLaM cells with PAM at 500 and $1,000 \mu \mathrm{M}$ in vitro consistently 
A

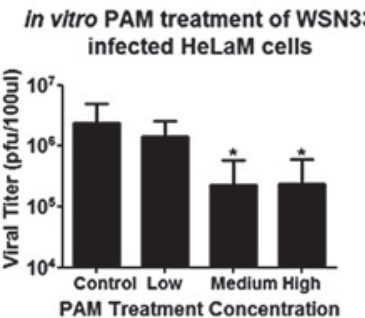

D

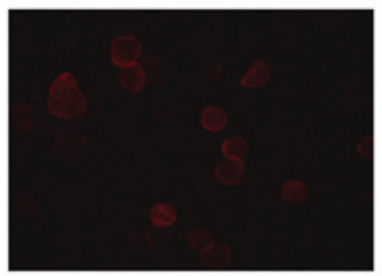

Infected treated
B

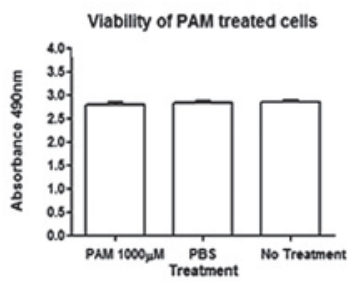

C

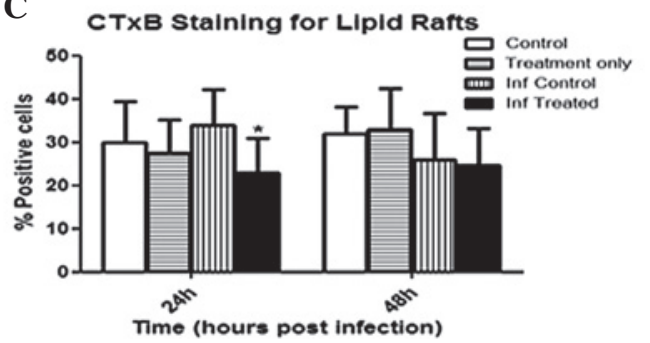

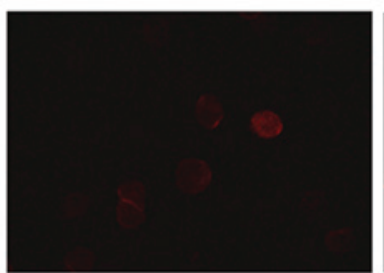

Uninfected treated

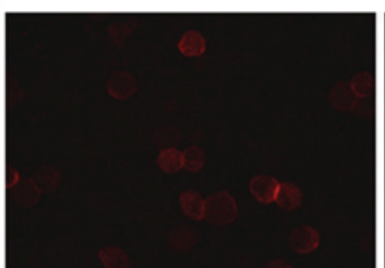

Infected untreated

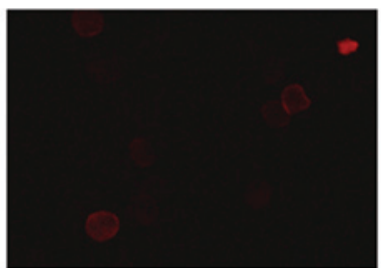

Control

Figure 1. In vitro PAM treatment of influenza virus-infected HeLaM cells. (A) Viral titers following treatment with low (100 $\mu \mathrm{M})$, medium (500 $\mu \mathrm{M})$ and high $(1,000 \mu \mathrm{M})$ concentrations of PAM ( $\mathrm{n}=5$ each). A significant reduction in viral titer of $\sim 1 \log (\mathrm{P}<0.05)$ was observed for cells treated with medium and high PAM concentrations. (B) No difference in cell viability was observed between the cells treated with the high PAM concentration and the control cells ( $\mathrm{n}=3$ each). (C) CTxB staining for quantification of lipid rafts in HeLa cells. A significant reduction $\left({ }^{*} \mathrm{P}<0.05\right)$ in $\mathrm{CTxB}$ staining was observed in the PAM-treated, influenza-infected cells compared with that in the untreated control cells after 24 h, but not after 48 h. (D) Representative immunofluorescence images of CTxB-stained cells displaying lower staining intensity in PAM-treated cells than in untreated cells. PAM, disodium pamidronate; WSN33, A/H1N1/WSN/1933; CTxB, cholera toxin subunit-B.

reduced viral titers by $\sim 1 \log$ compared with the titer in untreated infected control cells (Fig. 1A), suggesting a mechanism through FPPS inhibition. However, there was no further reduction in the viral titer of $>1 \log$ at PAM concentrations of $>500 \mu \mathrm{M}$. Using the MTS assay, no significant differences were observed between the cell viability upon PAM treatment and the viability in PBS-treated or untreated controls (Fig. 1B), indicating that PAM treatment did not cause deleterious effects in the cells in vitro.

PAM treatment reduces lipid raft formation in HeLaM cells. To ascertain whether the reduction in viral titer was due to lipid raft reduction by PAM treatment, staining for CTxB protein in cells treated with $1,000 \mu \mathrm{M}$ PAM in comparison with untreated cells was conducted. As shown in Fig. 1C and $\mathrm{D}$, there was a reduction in the number of $\mathrm{CTxB}$-positive cells in the infected and PAM-treated group compared with that in the infected but untreated group at $24 \mathrm{~h}$ post-infection, whereas no significant difference was observed between the two groups at $48 \mathrm{~h}$ post-infection. There were also no differences between uninfected cells subjected to different treatments at all time-points. These findings suggest that PAM treatment did not reduce existing lipid rafts, but prevented the increased lipid raft production required for influenza virus budding, which culminated in the decreased viral titer. The promising finding that PAM-mediated reduction of lipid raft production inhibited influenza replication in vitro warranted further investigations in vivo.

PAM treatment does not confer protection against influenza challenge in mice. To assess the efficacy of PAM treatment in vivo, infected mice were subjected to $\mathrm{PRO}$ and $\mathrm{PEP}$ regimes. Compared with PAM treatment studies of other infections (21), the PAM dosage was decreased to take into account direct delivery into the lungs. More frequent dosing was conducted in the PEP regime to achieve a higher PAM concentration in the animals than in the animals treated with the PRO regime.

Despite the PAM treatment of mice subjected to lethal influenza challenge, there were no significant differences in the Kaplan-Meier survival analyses (Fig. 2A), with mortality rates of $36.3 \%$ for the treated mice versus $54.5 \%$ for the untreated mice in the PRO regime; and $100 \%$ for the treated mice versus $100 \%$ for the untreated mice in the PEP regime. Similar weight loss patterns were observed with both regimes across all days (Fig. 2B). The higher mortality rates with the PEP regime may be attributed to the intratracheal treatment procedures following infection of the mice. Therefore, PAM treatment may not be efficacious in protecting against influenza challenge in vivo, in contrast to the in vitro observations.

PAM treatment does not significantly affect viral titer or immune gene expression in infected lungs. There were no observable differences in survival between PAM-treated and untreated mice post-influenza challenge. Therefore, it was investigated if there were any differences at the tissue and molecular levels in the animals. The viral titer in the lungs at day five post-infection remained unchanged following PAM treatment using either treatment regime (Fig. 3A). In contrast to the in vitro studies, PAM treatment failed to achieve a reduction in the viral titer in vivo, implying that its antiviral mechanism may not be useful in an in vivo setting.

qPCR analyses demonstrated that there were virtually no changes in the immune gene expression profiles between the treated and untreated mice infected with influenza virus (Fig. 3B). However, the only significant difference was elevated MyD88 expression levels in the PEP treatment group. Overall, PAM treatment in vivo did not cause major changes in the immune gene expression of infected lungs. 

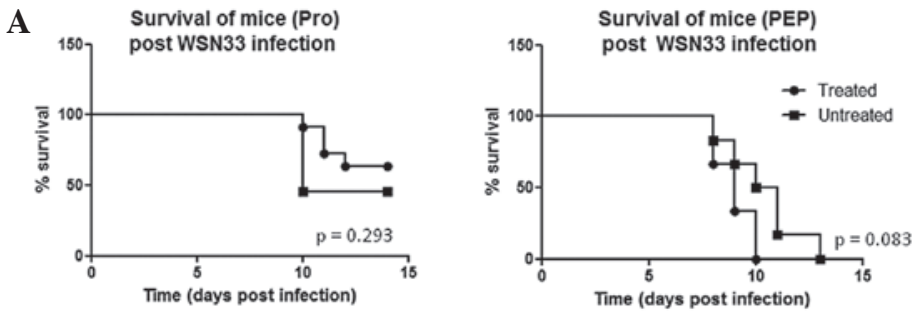

B Daily Weight post infection (Pro)

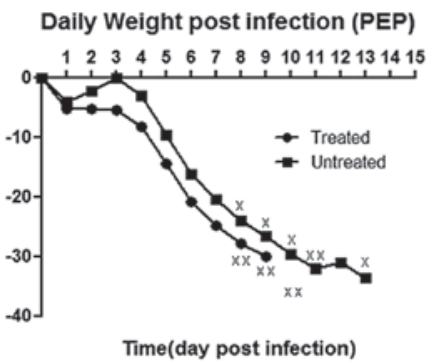

Figure 2. Survival analyses and weight loss profiles of influenza-infected mice with PAM treatment. (A) Kaplan-Meier survival curves for PRO (n=11) and PEP $(n=6)$ treatment regimes showing no significant difference in mortality between PAM-treated and untreated mice. Mice in the PEP regime died slightly earlier but this was not statistically significant. (B) There was no significant difference in weight loss patterns between the two groups of mice. X represents a single mouse euthanized at the stipulated end-point. PRO, prophylactic; WSN33, A/H1N1/WSN/1933; PEP, post-exposure prophylaxis; PAM, disodium pamidronate.

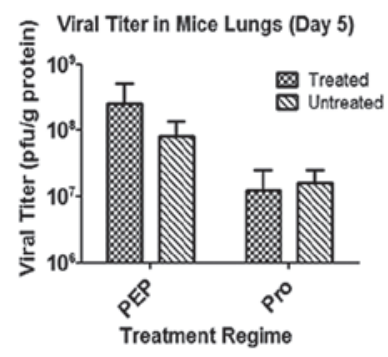

B

Immune gene expression at day 5 post infection (PEP)

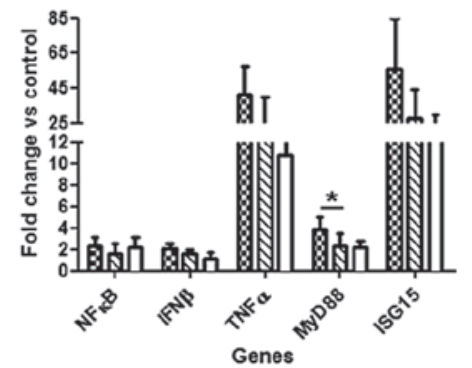

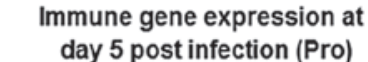

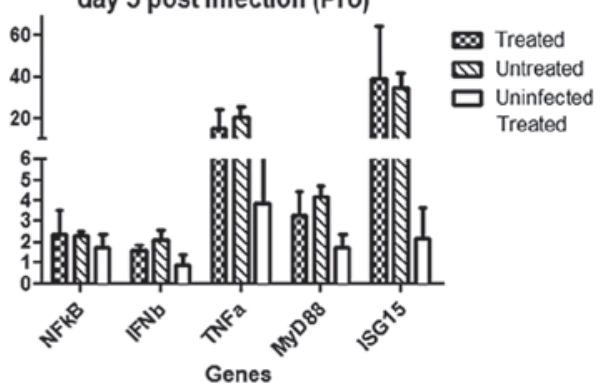

Figure 3. Viral and qPCR analyses of the lungs of mice treated with PAM. (A) There was no significant difference between the viral titers in the lungs of mice treated with each regime ( $\mathrm{n}=5$ each). (B) Expression profiles of immune and inflammatory genes in the lungs of mice ( $\mathrm{n}=5$ per group) were generally similar for the two treatment regimes, although MyD88 expression in the PEP regime was slightly elevated ( $\mathrm{P}<0.05)$. PRO, prophylactic; PEP, post-exposure prophylaxis; PAM, disodium pamidronate.

A

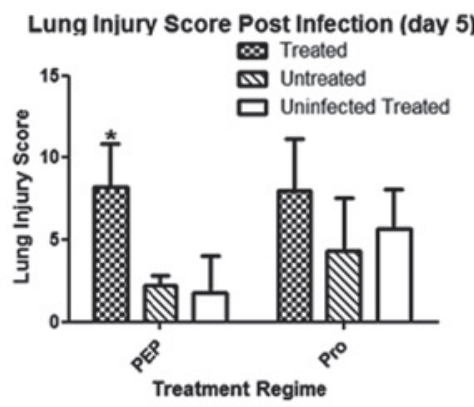

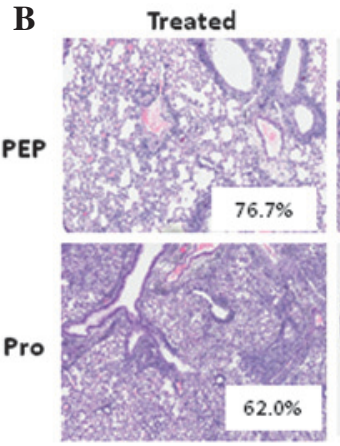
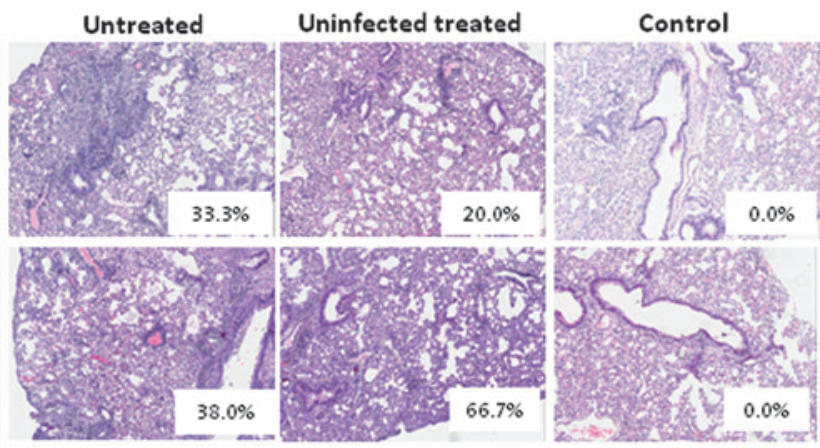

Figure 4. Histopathologic scoring of murine pulmonary injury. (A) Semi-quantitative scores ( $\mathrm{n}=3$ each) revealed augmented lung damage following the PEP regime $(\mathrm{P}<0.05)$, suggesting some detrimental effects of the treatment. $(\mathrm{B})$ Representative lung sections exhibit more diffuse damage in the two treatment regimes, probably due to adverse effects of FPPS inhibition in vivo. PRO, prophylactic; PEP, post-exposure prophylaxis; FPPS, farnesyl pyrophosphate synthase.

Histopathological studies of lung sections involving semi-quantitative scoring revealed that the lung injury scores were not significantly different between the infected groups in the PRO regime. Notably however, the PEP regime resulted in a significantly higher lung injury score in the PAM-treated group (Fig. 4A). The increased lung injury was largely attributed to the more pronounced and widespread areas of damage in the lungs (Fig. 4B). It was hypothesized that one mechanism 
of PAM treatment may be enhancement of the permeability of the lung vasculature, allowing the inflammation to be more diffuse in the lungs. Hence, the in vivo mechanism of PAM necessitates further investigation prior to exploitation as a potential therapy against infections that utilize lipid rafts, such as influenza.

\section{Discussion}

The major motivation of this study was to assess whether a small molecule compound could be effectively utilized in the treatment of influenza for greater cost effectiveness and ease of delivery compared with recombinant proteins such as viperin. Therefore, the present study evaluated the efficacy of a known chemical inhibitor of FPPS based on the hypothesis that its effects may be similar to those of viperin in controlling influenza infection.

Notably, the in vitro experiments revealed a reduction of lipid raft formation in response to the drug treatment of infected cells, whereas the lipid rafts of uninfected cells were not affected by the treatment. This suggests that PAM prevented an increase in lipid raft production by interfering with FPPS enzymatic activity. The kinetics of CTxB staining further suggest that the significant reduction in viral titer may be attributed to delayed lipid raft production following the treatment of infected cells compared with that in the untreated control cells. Further reduction was not observed with increasing PAM dosage, possibly due to the complex influenza virus budding process, i.e. budding stages where lipid rafts promote bud initiation and growth but inhibit bud excision and release (14). This finding thus suggests the potential use of conventional FFPS inhibitors to inhibit pathogens that require lipid raft formation as part of their life cycles (21).

By contrast, however, the in vivo results of the present study were not congruent with the hypothesis, implying that the direct delivery of PAM into the lungs may create a suboptimal environment for influenza virus replication. The prophylactic and therapeutic regimens that were tested did not significantly alter the susceptibility of mice to the infection, nor did they decrease viral titers in the lungs; however, they unexpectedly increased lung injury in the mice.

These in vivo findings appear to be similar to those in the viperin knockout mouse model (19), where despite viperin efficacy in vitro against influenza infection, the importance of the protein and its mechanism appears to be negated by other factors in vivo, as demonstrated by the equal susceptibility of wild-type and knockout mice to influenza infection. In the present study, similar results were obtained by mimicking the mechanism of viperin instead of using the knockout phenotype. The effects of the intervention appeared to have been negated by the more complex scenario in vivo, where even repeated doses of PAM did not produce the desired effect.

One possible explanation is that the treatment interferes with cholesterol synthesis by epithelial and endothelial cells in the lungs, thus affecting their membrane integrity and causing greater 'leakiness', allowing the infection site to be more extensive. This was demonstrated by the more diffuse extent of lung injury in the PAM-treated mice. Such damage may arise from the leakage from the vasculature into the lungs, culminating in cell death.
Another possible explanation may be the drug's ability to activate $\gamma \delta$ T-cells in an acute phase response in infected mice, which aggravates the inflammation (22). PAM confers a protective effect through the activation of $\gamma \delta \mathrm{T}$-cells when administered via the intraperitoneal route in a humanized mouse model (23). The greater magnitude of inflammation arising from the direct activation of $\gamma \delta$ T-cells in the lungs may augment lung damage, as observed by the more diffused damage and higher lung injury score in the PAM-treated mice in the present study.

In conclusion, although PAM effectively diminished influenza virus titers in vitro, this molecular inhibitor of FPPS lacked efficacy in alleviating influenza-induced pulmonary damage in vivo. Nevertheless, this study indicated the potential of a clinically approved FPPS inhibitor as a strategy for controlling influenza infection, by targeting lipid rafts which are required for the viral budding and release process. The administration of this drug may be further refined to allow its optimal retention in infected tissues, or PAM may be tested in other infections that are more susceptible to FPPS inhibition, such as West Nile virus (24).

\section{Acknowledgements}

The authors thank H.M. Shen, Y. Shi, Connie Foo, S.H. Lau, N. Li, A.N. Moorthy and W.P. Poh for their assistance. This study was supported by the National University of Singapore, the National Research Foundation (Singapore) and the Singapore-Massachusetts Institute of Technology Alliance for Research and Technology.

\section{References}

1. Poland GA, Jacobson RM and Targonski PV: Avian and pandemic influenza: an overview. Vaccine 25: 3057-3061, 2007.

2. de Wit E and Fouchier RA: Emerging influenza. J Clin Virol 41: $1-6,2008$

3. Memoli MJ, Morens DM and Taubenberger JK: Pandemic and seasonal influenza: therapeutic challenges. Drug Discov Today 13: 590-595, 2008.

4. Rothberg MB, Haessler SD and Brown RB: Complications of viral influenza. Am J Med 121: 258-264, 2008.

5. Watanabe T, Watanabe S and Kawaoka Y: Cellular networks involved in the influenza virus life cycle. Cell Host Microbe 7: 427-439, 2010.

6. Karlas A, Machuy N, Shin Y, et al: Genome-wide RNAi screen identifies human host factors crucial for influenza virus replication. Nature 463: 818-822, 2010.

7. Stertz S and Shaw ML: Uncovering the global host cell requirements for influenza virus replication via RNAi screening. Microbes Infect 13: 516-525, 2011.

8. König R, Stertz S, Zhou Y, et al: Human host factors required for influenza virus replication. Nature 463: 813-817, 2010.

9. Fitzgerald KA: The interferon inducible gene: Viperin J Interferon Cytokine Res 31: 131-135, 2011.

10. Gerber SA and Pober JS: IFN-alpha induces transcription of hypoxia-inducible factor-1alpha to inhibit proliferation of human endothelial cells. J Immunol 181: 1052-1062, 2008.

11. Wang X, Hinson ER and Cresswell P: The interferon-inducible protein viperin inhibits influenza virus release by perturbing lipid rafts. Cell Host Microbe 2: 96-105, 2007.

12. Simons K and Ehehalt R: Cholesterol, lipid rafts, and disease. J Clin Invest 110: 597-603, 2002.

13. Reilly JF, Martinez SD, Mickey G and Maher PA: A novel role for farnesyl pyrophosphate synthase in fibroblast growth factor-mediated signal transduction. Biochem J 366: 501-510, 2002.

14. Nayak DP, Balogun RA, Yamada H, Zhou ZH and Barman S: Influenza virus morphogenesis and budding. Virus Res 143: 147-161, 2009. 
15. Ono A and Freed EO: Role of lipid rafts in virus replication. Adv Virus Res 64: 311-358, 2005.

16. van Beek E, Pieterman E, Cohen L, Löwik C and Papapoulos S: Farnesyl pyrophosphate synthase is the molecular target of nitrogen-containing bisphosphonates. Biochem Biophys Res Commun 264: 108-111, 1999.

17. Kavanagh KL, Guo K, Dunford JE, et al: The molecular mechanism of nitrogen-containing bisphosphonates as antiosteoporosis drugs. Proc Natl Acad Sci USA 103: 7829-7834, 2006.

18. Guo RT, Cao R, Liang PH, et al: Bisphosphonates target multiple sites in both cis- and trans-prenyltransferases. Proc Natl Acad Sci USA 104: 10022-10027, 2007.

19. Tan KS, Olfat F, Phoon MC, et al: In vivo and in vitro studies on the antiviral activities of viperin against influenza H1N1 virus infection. J Gen Virol 93: 1269-1277, 2012.

20. Matute-Bello G, Winn RK, Jonas M, Chi EY, Martin TR and Liles WC: Fas (CD95) induces alveolar epithelial cell apoptosis in vivo: implications for acute pulmonary inflammation. Am J Pathol 158: 153-161, 2001.
21. Yardley V, Khan AA, Martin MB, et al: In vivo activities of farnesyl pyrophosphate synthase inhibitors against Leishmania donovani and Toxoplasma gondii. Antimicrob Agents Chemother 46: 929-931, 2002.

22. Rossini M, Adami S, Viapiana O, et al: Long-term effects of amino-bisphosphonates on circulating $\gamma \delta \mathrm{T}$ cells. Calcif Tissue Int 91: 395-399, 2012.

23. Tu W, Zheng J, Liu Y, et al: The aminobisphosphonate pamidronate controls influenza pathogenesis by expanding a gammadelta $\mathrm{T}$ cell population in humanized mice. J Exp Med 208: 1511-1522, 2011.

24. Szretter KJ, Brien JD, Thackray LB, Virgin HW, Cresswell P and Diamond MS: The interferon-inducible gene viperin restricts West Nile virus pathogenesis. J Virol 85: 11557-11566, 2011. 